\title{
Bursts detected in hard X-rays by the IBIS telescope onboard the INTEGRAL observatory in 2003-2007
}

\section{I.V. Chelovekov}

Space Research Institute of the Russian Academy of Sciences

E-mail: chelovekov@hea.iki.rssi.ru

\section{S.A. Grebenev}

Space Research Institute of the Russian Academy of Sciences

E-mail: sergei@hea.iki.rssi.ru

\begin{abstract}
All of the observations performed with the IBIS telescope onboard the INTEGRAL observatory during the first 4.5 years of its in-orbit operation have been analyzed to find X-ray bursts. The time history of the IBIS/ISGRI total count rate in the energy range 15-25 keV revealed several hundreds of bursts with a duration of 5-500 s at high statistical significance level (over the entire period of observations, only one event could be detected by chance with a probability of $20 \%$ ). In addition to the events associated with cosmic gamma-ray bursts (detected in the field of view or the ones that passed through the IBIS shield), solar flares, activity of the soft gamma repeater SGR 1806-20 and several other sources, we were able to localize 424 type I X-ray bursts and, with three exceptions, to identify them with previously known X-ray bursters. The three exceptions were: a burst from a new burster in a low X-ray intensity state that received the name IGR J173642711/17380-3749, GRB 060428C, and a burst from AXJ1754.2-2754, which let us to identify this source as an X-ray burster. Curiously enough, more than 300 bursts were detected from one X-ray burster, GX 354-0. The statistical distributions of bursts in duration, peak flux, fluence, preburst persistent flux and recurrence time have been analyzed for this source. Some of the bursts observed with the IBIS/ISGRI telescope were also detected by the JEM-X telescope onboard the INTEGRAL observatory in the standard X-ray energy range 3-20 keV.
\end{abstract}

7th INTEGRAL Workshop

September 8-11 2008

Copenhagen, Denmark

* Speaker. 


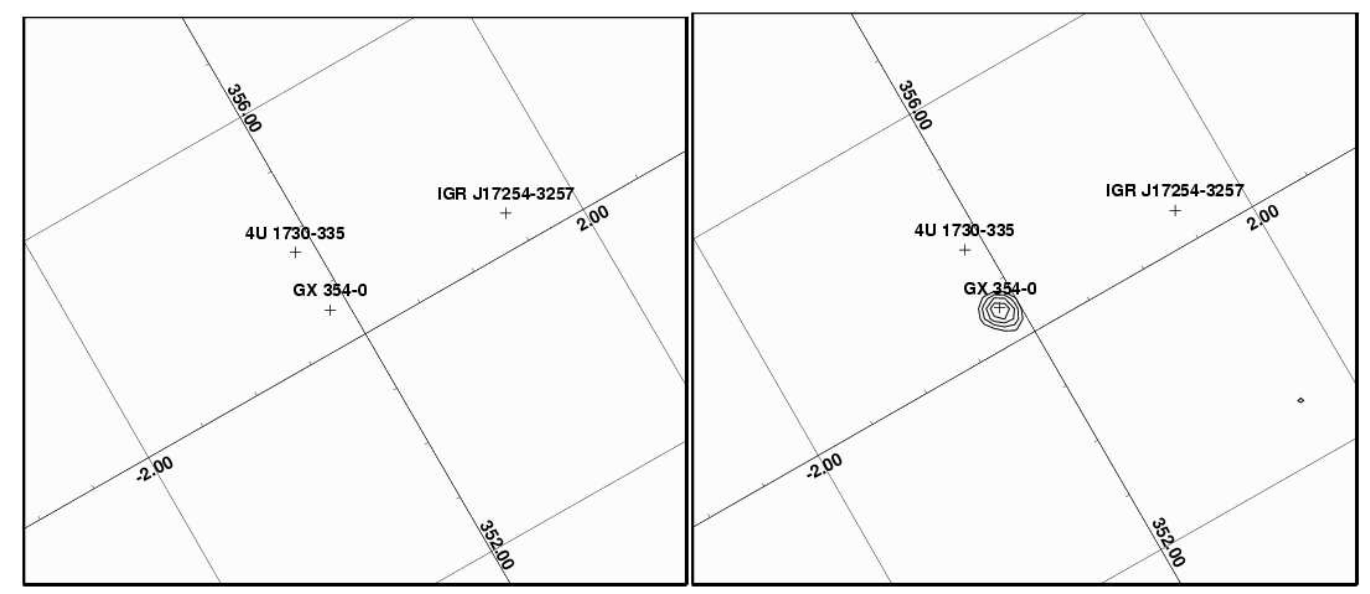

Figure 1: Images of the sky region in the IBIS/ISGRI field of view $10 \mathrm{~s}$ before the onset of the burst detected from GX 354-0 on September 20, 2003 (left panel) and at its maximum (right panel). In both cases the exposure is $5 \mathrm{~s}$ and the energy band is $15-25 \mathrm{keV}$. The contours indicate the region of reliable detection of the source at the signal-to-noise ratio $\mathrm{S} / \mathrm{N}=4,6.5,9.1,11.7$, and 14.3 standard deviations (given with a logarithmic step of 1.22).

\section{INTRODUCTION}

The bursts of different types are observed in hard X-rays (15-25 keV in our case). Among them are cosmic gamma-ray bursts, solar flares, events from magnetars, etc. Type I X-ray bursts are the most interesting and fruitful of them. The detection of type I X-ray bursts is together with the detection of coherent X-ray pulsations the strongest argument for the presence of a neutron star in a binary system.

According to our understanding of their origin, type I X-ray bursts result from unstable thermonuclear burning of $\mathrm{H} / \mathrm{He}$ on the surface of neutron stars in Low Mass X-ray Binaries (LMXBs). Due to the accretion of matter from an optical star onto a neutron star, we can see a quasi-persistent $\mathrm{X}$-ray emission from the system. Note that this persistent emission may be bellow detection limit of X-ray observations in hard X-ray band (e.g. INTEGRAL). The accreted matter accumulated on the surface of the neutron star gives a birth to a type I X-ray burst when the density and temperature in the foundation of accumulated layer are high enough unstable $\mathrm{H} / \mathrm{He}$ burning starts, consuming all the fuel within several seconds to several minutes (depending on the burning regime, see e.g. [1]). The enormous amount of energy released during the explosion makes the system luminosity rise in a couple of orders of magnitude for a short time, making even a persistently weak source well detectable.

Such a source could either be detected in a deep survey of the sky, but then no indication or hint of its nature will be available, or during a type I X-ray burst that would identify it as a new $\mathrm{X}$-ray burster, that is a NS in a LMXB.

\section{DATA ANALYSIS}

To fi nd X-ray bursts in this research we used data from the ISGRI detector of the IBIS telescope on-board INTEGRAL. Most of the energy during a type-I X-ray burst is emitted below 15-25 keV, 
so ISGRI might not be the best detector for research of this kind - not all of the bursts might have hard tails strong enough to be signifi cantly detected in the 15-25 keV energy band, that we used. But this disadvantage actually became an advantage, allowing us to study a sample of hard X-ray bursts and discover a unique hard X-ray burster - GX 354-0 (see below). There are also several advantages to do this research with IBIS: it has a huge fi eld of view; it is an imaging telescope and lets one localize and identify the source responsible for the burst; and fi nally it dedicates a bulk of the observing time to the Galactic Center, where most of the elder stellar population of the Galaxy (including Low Mass X-ray Binaries - ones of interest to us) is situated.

Our procedure to search for bursts was the following: fi rst, we analysed the count rate history from the whole ISGRI detector selecting the statistically signifi cant excesses in the count rate; second, we reconstructed the images of the sky inside the fi eld of view of the telescope (for this purpose we used a standard OSA software package) for the $5 \mathrm{~s}$ long time bins (most of type I Xray bursts last longer than that) during the detected excess (Fig. 1, right panel). Comparing the statistical signifi cance of detection of each of the revealed sources with the image, obtained for merely time interval, corresponding to normal not excessive count rate (Fig. 1, left panel), it is easy to identify the source responsible for the detected burst.

We performed this kind of analysis for all the IBIS data from February 2003 to March 2007 which is around 40000 Science Windows and around 100 Ms exposure.

\section{RESULTS}

\subsection{Catalog of type I X-ray bursts}

Among all the bursts, detected by the IBIS telescope and revealed within this research our special attention was focused onto type I X-ray bursts. As a result we constructed a catalog of all type I X-ray bursts, detected by IBIS telescope in a hard (15-25 keV) X-ray band from February 2003 to March 2007. The catalog consists of 422 bursts from 17 known bursters an two bursts from newly discovered bursters. The catalog will soon be available through RSDC web page (http://hea.iki.rssi.ru/ru/index.php?page=bursters).

\subsection{Discovery of a new X-ray burster: IGR J17364-2711/17380-3749}

The main goal of this work was to discover new X-ray bursters with weak persistent emission. One of such bursters has been actually discovered - IGR J17364-2711/17380-3749. The burst from this previously unknown source was detected by the IBIS telescope on Feb. 17, 2004 (see [2],[3]). The burst flux reached a peak value of 1.85 Crab at 14:41:30 (UTC). Fig. 2 shows maps of the statistical signifi cances of the source detection constructed for a 10-s time interval during the burst (left panel) and over the whole INTEGRAL pointing exposure (right panel).

The duration of the burst was $13 \mathrm{~s}$ (in $15-25 \mathrm{keV}$ energy band) with a 5-s rise and an 8-s decay. Unfortunately the telescope was pointed around $7^{\circ}$ away from this source at the moment of the burst, so we could not use the X-ray telescope aboard INTEGRAL (JEM-X) to study it in the standard X-ray energy band.

The burst recurrence time for the new source was estimated to be:

$$
\tau=\alpha T F / F_{X} \simeq 6 \times 10^{8} \mathrm{~s} \simeq 20 \text { years } .
$$




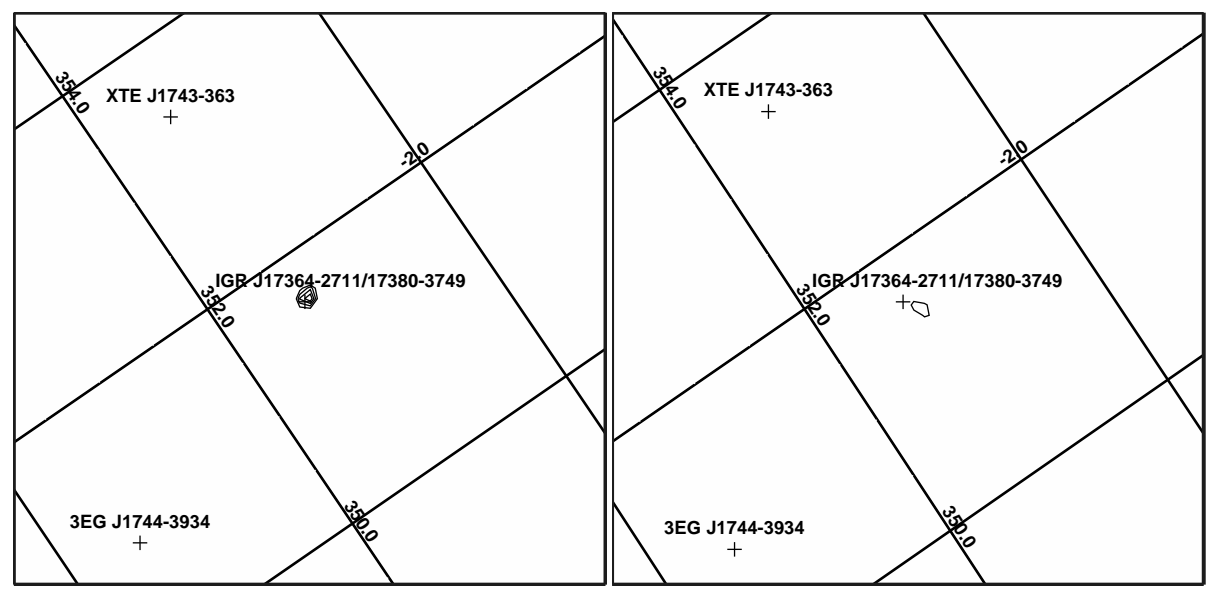

Figure 2: Images of the sky region in the IBIS/ISGRI field of view obtained on February 17, 2004 (left panel) during the burst detected from the hitherto unknown burster IGR J17364-2711/17380-3749 and (right panel) during the entire observing session (except the burst time). The exposures were $10 \mathrm{~s}$ and more than $93 \mathrm{ks}$ respectively. Both images were obtained in the $15-25 \mathrm{keV}$ energy band. The contours are given for $\mathrm{S} / \mathrm{N}=6,6.7,7.5,8.2,9.0$ standard deviations.

Here $\alpha \sim 100$ is a factor that shows the effi ciency of the energy release via accretion compared to the energy release via thermonuclear burning on the neutron star's surface. We assumed all bursts from this source similar to the burst detected by the ISGRI detector, i.e., that they have a duration $T \simeq 13$ and a maximum flux $F \simeq 1.6 \mathrm{Crab}$, and we took the persistent photon flux from the source to be $F_{X} \simeq 3 \times 10^{-6} \mathrm{Crab}$ in the energy range $15-25 \mathrm{keV}$ (that corresponds to the 2-30$\mathrm{keV}$ luminosity $L_{X} \simeq 1 \times 10^{33} \mathrm{erg} \mathrm{s}^{-1}$ at a distance of $8.5 \mathrm{kpc}$ for a spectrum similar to that of the Crab Nebula, typical for the "off" state luminosity of low-mass X-ray binaries). The detection of only one such burst in $\sim 13 \mathrm{Ms}$ of observations implies that there can be no more than $\sim 50$ such binaries in this Galactic region, where we simultaneously observe up to $80 \%\left(4 \times 10^{9} M_{\odot}\right.$ in an $\sim 8^{\circ} \times 8^{\circ}$ fi eld) of the Galactic bulge stars, not counting the stars $\left(3 \times 10^{9} M_{\odot}\right)$ of other Galactic components (see [6]).

\subsection{Identification of AX J1754.2-2754 as an X-ray burster}

One of the bursts, discovered during our research (see [4]), originated from the known, but weak and, poorly studied persistent source AX J1754.2-2754 (Fig. 3). The burst was recorded on April 16, 2005, by both JEM-X and IBIS telescopes. Analysis of its time profi les and spectra allows this event to be attributed to a type I X-ray burst and the source itself identifi ed as an X-ray burster. Peculiarities of the X-ray emission observed at the initial phase of the burst pointed to an expansion and a corresponding cooling of the neutron star photosphere that took place at this time under the action of radiation pressure. Assuming the luminosity of the source at this phase to be the Eddington one, we have estimated a distance to the source $d=6.6 \pm 0.3 \mathrm{kpc}$ (for a hydrogen atmosphere of the neutron star) and $d=9.2 \pm 0.4 \mathrm{kpc}$ (for a helium atmosphere).

\subsection{Discovery of GRB 060428C}

While analyzing the data we detected and localized a cosmic gamma-ray burst (Fig. 5) 


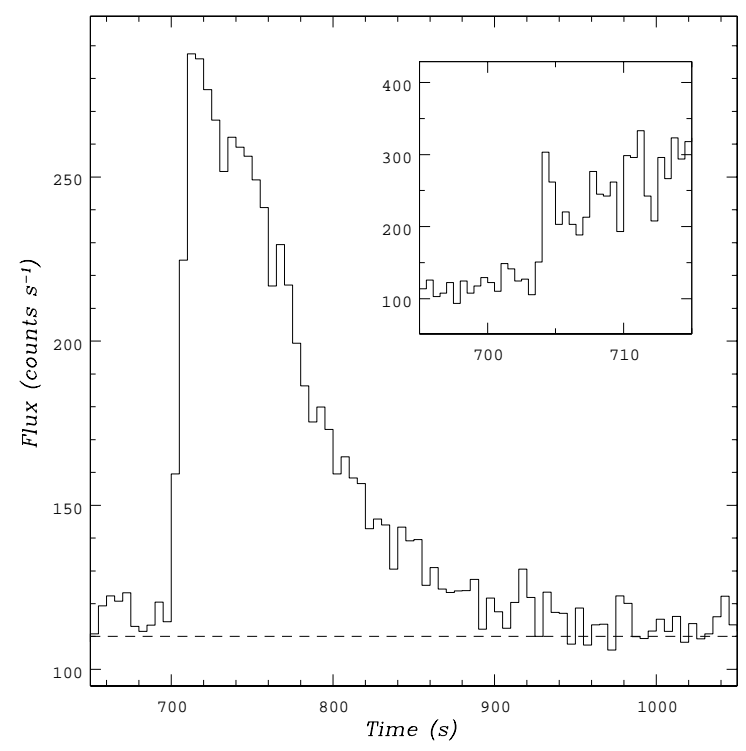

Figure 3: Count rate record by the JEM-X telescope on April 16, 2005, during the X-ray burst from AX J1754.2-2754 (the energy range is $3-20 \mathrm{keV}$, the time, in seconds, from the beginning of pointing at $21^{h} 58^{m} 35^{s} \mathrm{UT}$ is along the $\mathrm{X}$ axis, the time step is $5 \mathrm{~s}$ ). The curve was corrected for the detector dead time and for the coefficient related to the switch-on of a "gray" filter by the JEM-X electronics during the burst. The initial burst phase with a smaller time step (1 s), shows its complex structure with a possible precursor, is presented in the inset.

recorded on April 28, 2006, by IBIS and SPI (another telescope on-board INTEGRAL) telescopes in their fi elds of view (see [7]). Since the burst was not revealed by the INTEGRAL Burst Alert System (IBAS), information about its coordinates was not distributed in time and no search for its afterglow was performed. The burst was also recorded by the KONUS/WIND and RHESSI satellites. Its $20-200 \mathrm{keV}$ fluence was $2.3 \times 10^{-6} \mathrm{erg} \mathrm{cm}^{-2}$, the peak flux was $3.6 \times 10^{-7} \mathrm{erg} \mathrm{cm}^{-2} \mathrm{~s}^{-1}$ (3.9 phot. $\mathrm{cm}^{-2} \mathrm{~s}^{-1}$ ). The burst had a complex multi-peak profi le and stood out among typical bursts by an increase in its hardness with time. At the flux peak, the spectrum was characterized by a photon index $\alpha \simeq-1.5$ and a peak energy $E_{p} \simeq 95 \mathrm{keV}$. The burst lasted for $\sim 12 \mathrm{~s}$, after which its afterglow decaying as a power law with an index $\gamma \sim-4.5$ was observed at energies $15-45 \mathrm{keV}$. The spectral hardness decreased noticeably during the afterglow.

\subsection{Analysis of bursts from GX 354-0}

One of the main and most interesting results of this research is a fact that 313 bursts, which is almost $3 / 4$ of our sample of 424 bursts, were detected from the well known and stable X-ray burster GX 354-0. Table 1 shows that even though GX 354-0 was the most active bursting source during the 1996-2003 WFC/BeppoSAX survey, it was far from being as active as INTEGRAL detected (see [5]). The only difference between these BeppoSAX and INTEGRAL surveys seems to be the energy band (note, that the INTEGRAL's exposure dedicated to each source is a factor of 2-3 larger than that of BeppoSAX). To check the possibility of selection effects we constructed a distribution of angular distances from the center of the IBIS fi eld of view to several sources under consideration (Fig. 4). Each point on this plot corresponds to a single pointing of INTEGRAL and lasted $\sim 1$ 


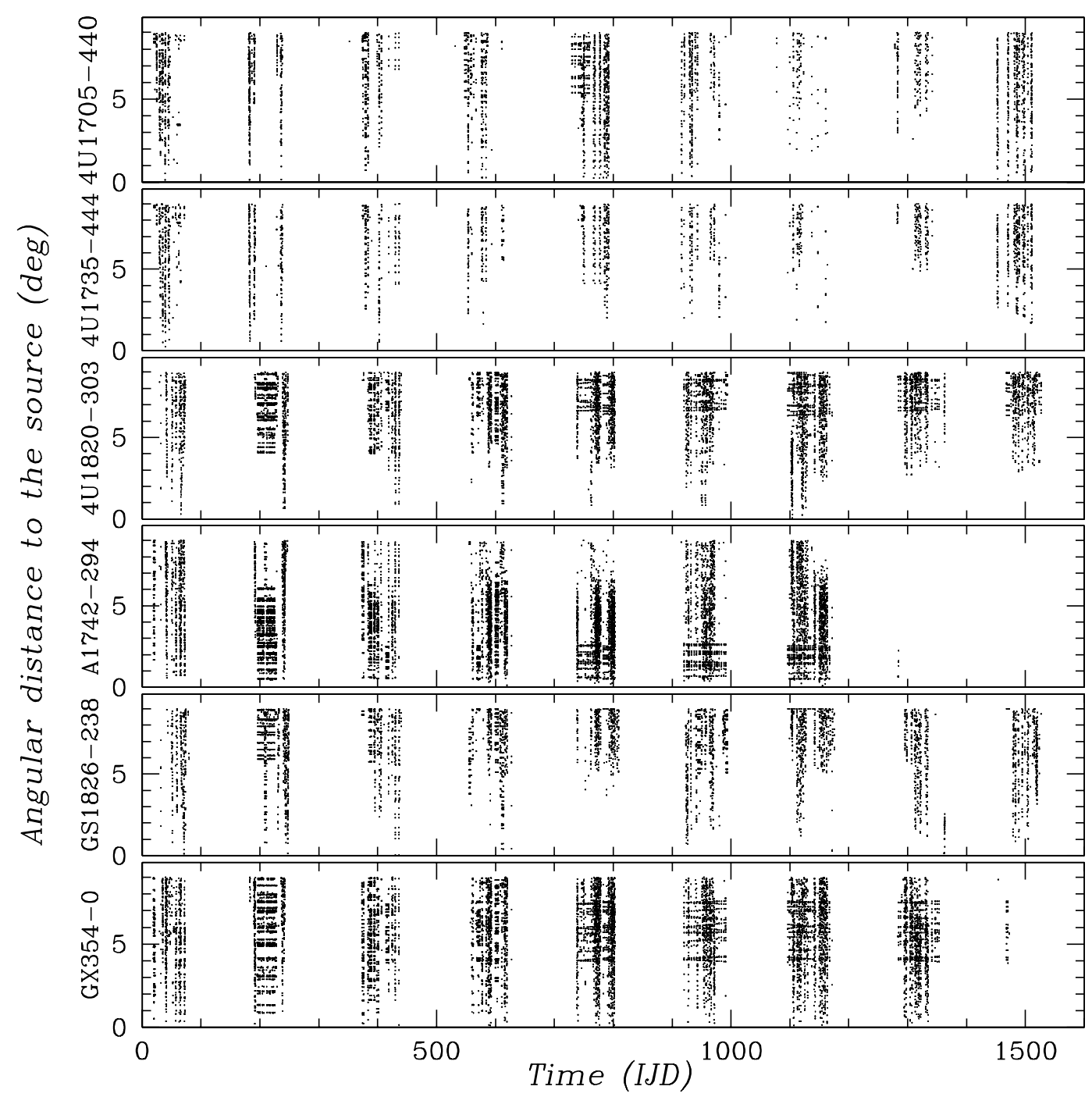

Figure 4: Angular distance from the center of the INTEGRAL field of view to several well known stable $\mathrm{X}$-ray bursters as a function of time (in INTEGRAL JD). Each point corresponds to a single INTEGRAL observation lasted $\sim 1$ hour.

hour, so the fi gure indicates an exposure, dedicated to each of the sources. Obviously GX 354-0 does not stand out generally. Thus we can state, that the detection of such a large number of bursts with signifi cant hard X-ray emission from GX 354-0 is not due to selection effects, but is a sign of some unique features of this source or its state.

Fig. 6 shows a distribution of 145 bursts (among the 313 burst sample from GX 354-0) that were also observed by the JEM-X telescope, as a function of their preburst luminosity. It is obvious, that most of the bursts are emitted while the source persistent luminosity was around $10^{37} \mathrm{erg} \mathrm{s}^{-1}$. At higher luminosities the number of bursts decreases, most likely due to a transition into a nonexplosive thermonuclear burning state. At lower luminosities it decreases simply due to the fact, that with the smaller luminosity (accretion rate) it takes more time to accrete enough matter for a burst to begin. Although GX 354-0 is far not the only source that has a persistent luminosity $\sim 10^{37}$ 


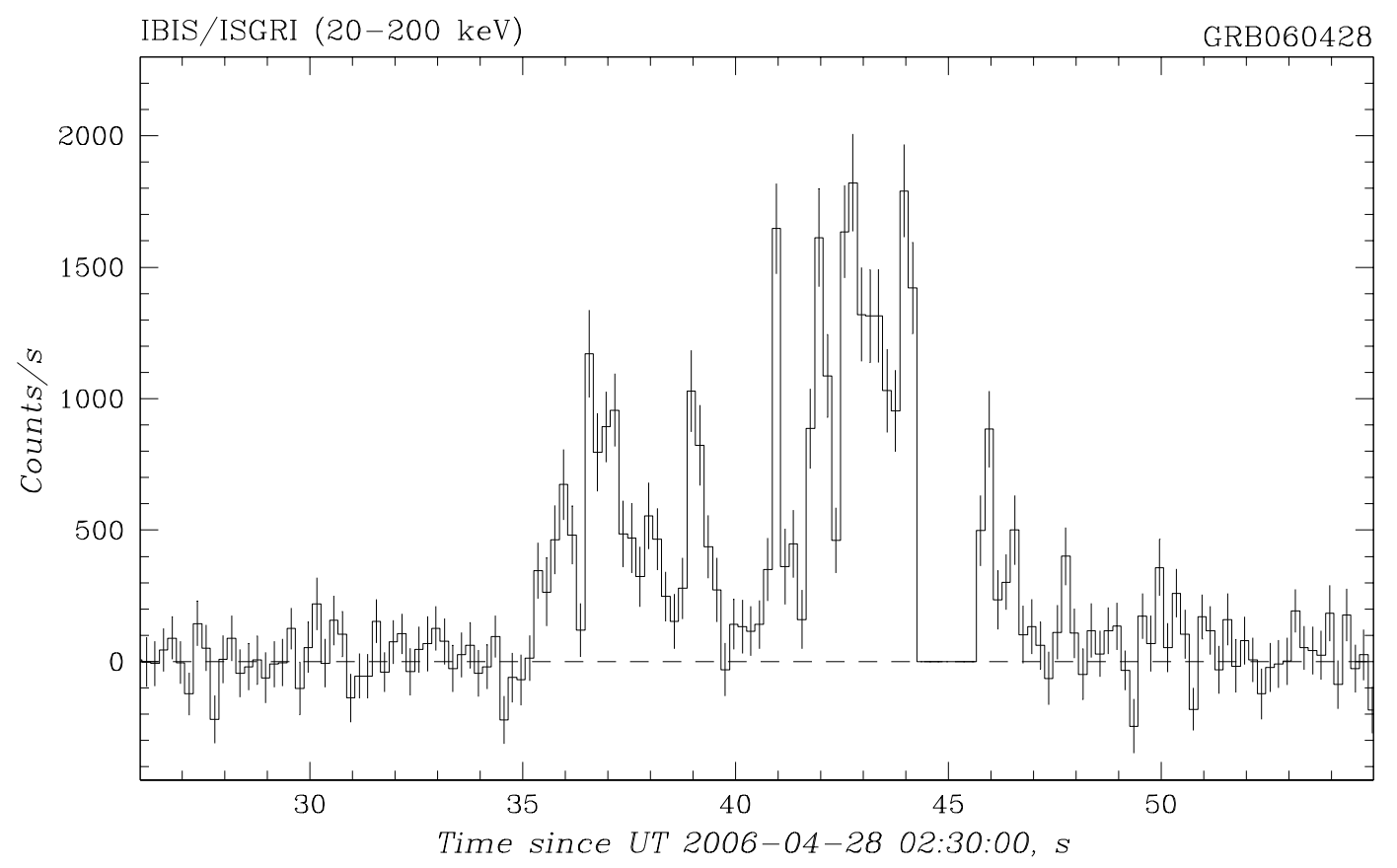

Figure 5: IBIS/ISGRI time profile for GRB $060428 \mathrm{C}$ in the energy band 20-200 keV. The time, in seconds, from $2^{h} 30^{m} 00^{s} \mathrm{UT}$ is along the $\mathrm{X}$ axis, the time step is $0.2 \mathrm{~s}$.

$\mathrm{erg} \mathrm{s}^{-1}$, it is the only one producing so many bursts, detectable in hard X-rays. The nature of this phenomena is yet to be resolved.

Fig. 7 shows distributions of 313 GX 354-0 bursts over their duration (left panel) and burst recurrence time (right panel). We can see that the shortest burst was $5 \mathrm{~s}$ long and the longest - $30 \mathrm{~s}$ long, while most of the bursts had a duration of 10-15 s. Duration of the burst is calculated using the $15-25 \mathrm{keV}$ detector lightcurve with $1 \mathrm{~s}$ time bin. The beginning and the end of a burst are taken at moments, when the count rate enhancement over the mean value is $10 \%$ from the peak one.

The burst recurrence time is the time necessary to accrete amount of matter large enough to trigger the burst. The recurrence time for most of the GX 354-0 bursts, as it follows of the fi gure, is $\sim 3-4$ hours. Any longer recurrence times present on this figure might be either true ones, or might simply represent double, triple or etc. recurrence time periods, for which it was impossible to detect the consequent burst due to interruption in the INTEGRAL observations.

The distribution of GX 354-0 bursts as a function of their fluence (for 145 bursts that were observed with both the IBIS and JEM-X telescopes) is shown in Fig. 8 (left panel). One may mention two peaks on the distribution at $\sim 4 \times 10^{38} \mathrm{erg}$ and $\sim 1 \times 10^{39} \mathrm{erg}$. It may represent two populations of bursts, that are different in duration and peak flux (see below). The right panel of the same fi gure shows the distribution of these bursts as a function of their peak luminosity. The interesting feature of this fi gure is a right peak, corresponding to the super Eddington luminosity for a $1.4 M_{\odot}$ neutron star, assuming solar abundance. Assuming the peak is due to the He burst, we estimated a mass of the neutron star in this binary system to be $M \simeq 1.7 \pm 0.7 M_{\odot}$.

Fig. 9 shows the burst recurrence time, duration, fluence and preburst luminosity versus the 


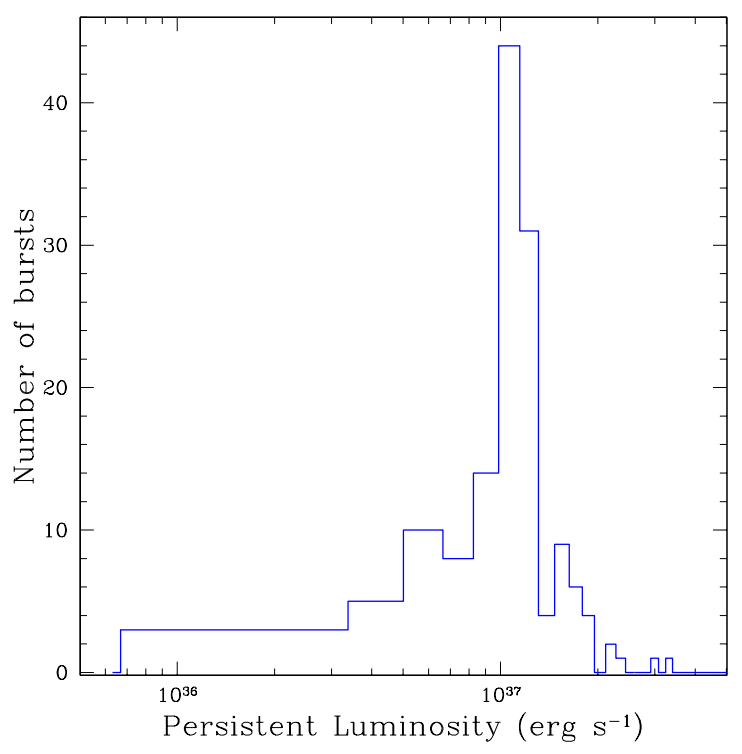

Figure 6: Distributions of 145 bursts detected from GX 354-0 by the JEM-X and IBIS telescopes in 20032007 vs. its preburst luminosity.

burst peak luminosity for 145 GX 354-0 bursts, detected by both the IBIS and JEM-X telescopes. While there seems to be no correlation between the recurrence time and peak luminosity, the other dependencies exhibit rather interesting behavior. The peak luminosity seems to be proportional to both the duration and the fluence of the burst. Fig. 10 (left panel) shows the proportionality between burst fluence and duration. Together these two proportionalities lead one to the following conclusion: the more energetic the bursts are, the longer they will be and the greater will be their peak luminosity. So not all of the bursts at all will reach the Eddington peak luminosity.
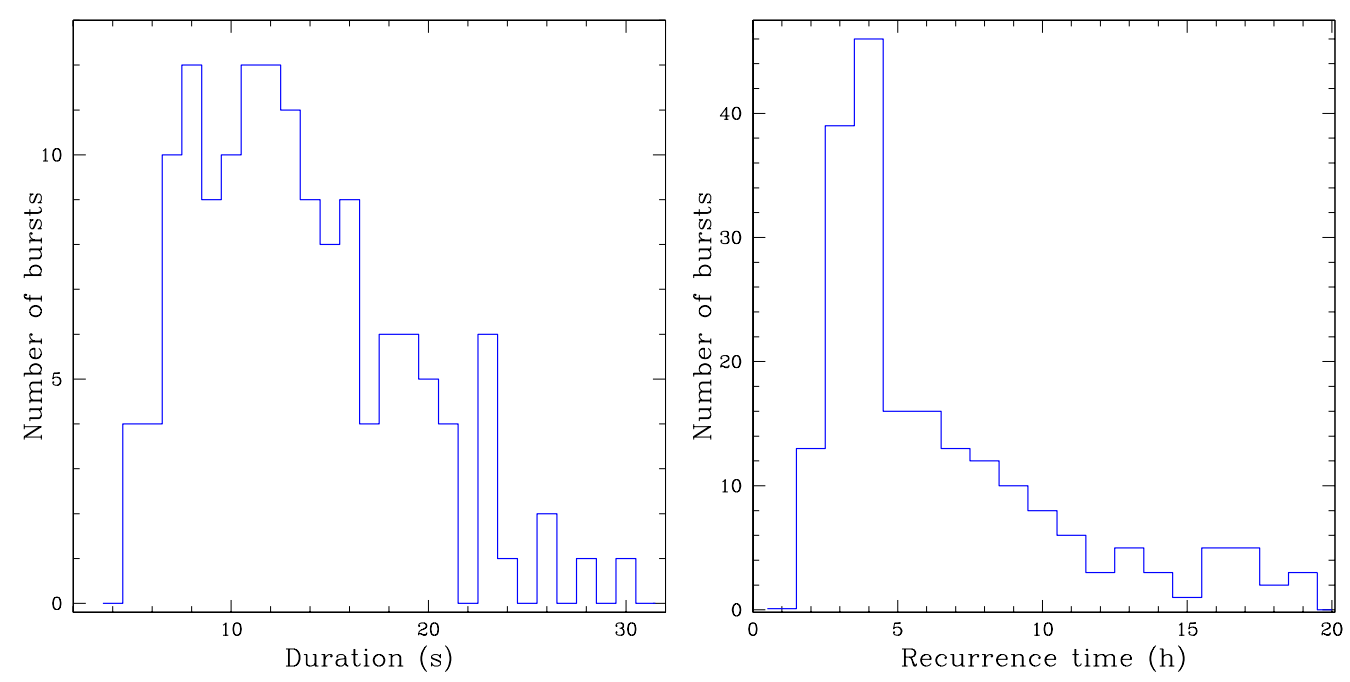

Figure 7: Distributions of all the bursts detected from GX 354-0 by the IBIS telescope in 2003-2007 vs. the burst duration (left panel) and burst recurrence time (right panel). 

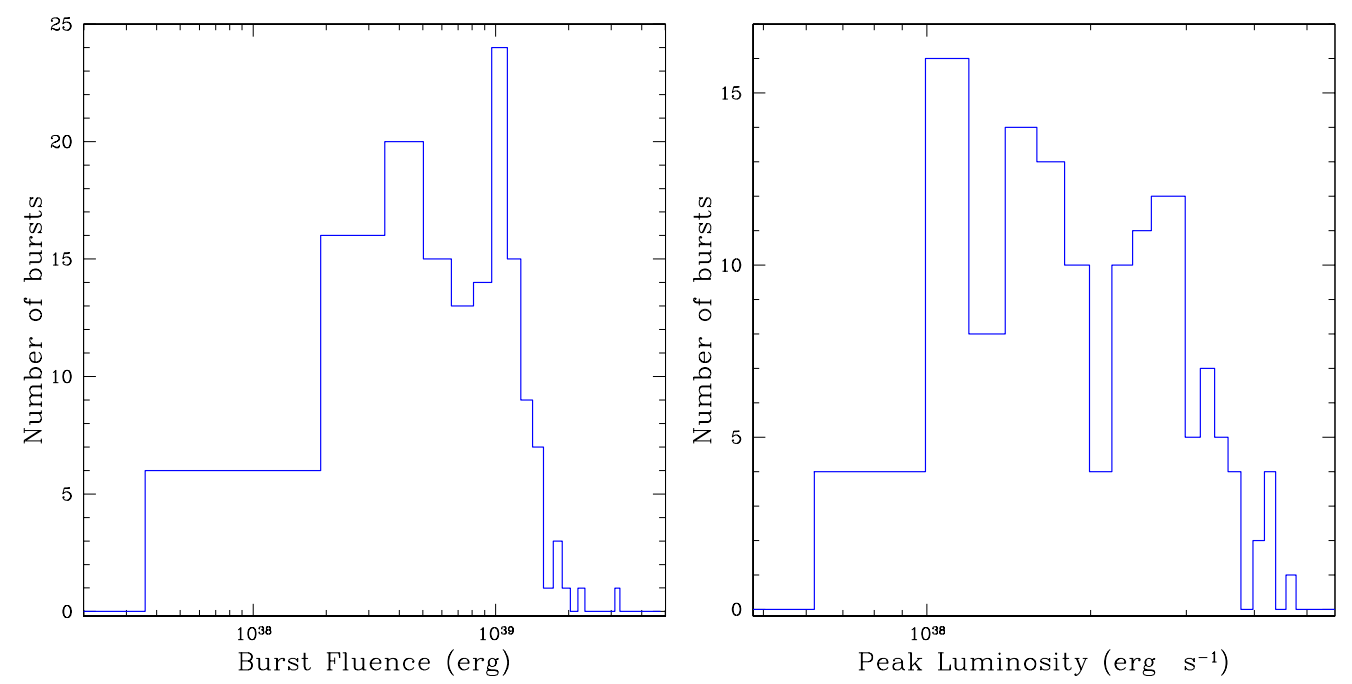

Figure 8: Distribution of 145 bursts detected from GX 354-0 by the JEM-X and IBIS telescopes in 20032007 vs. the burst fluence (left panel) and burst peak luminosity (right panel).

There is yet another trend in Fig. 9 and Fig. 10. The points seem to be randomly distributed, witnessing of no clear dependence between the quantities, however two "islands" - areas where about half of the points are situated can be seen on both figures. At almost the same levels of preburst persistent luminosity there seems to be two populations of bursts, which actually also differ in duration: shorter bursts with smaller fluence and peak luminosity, and longer ones with larger fluence and peak luminosity. The fact, that these two populations occupy the same preburst persistent luminosity level indicates that longer bursts either have a larger calm preburst period to accumulate more fuel or they burn the fuel left from the previous burst. We might witness here a transition between $\mathrm{H}$ triggered $\mathrm{He}$ bursts and $\mathrm{He}$ bursts in $\mathrm{H}$ poor environment ([8]).

Fig. 11 (left panel) shows the preburst persistent luminosity as a function of the burst recurrence time. These quantities should be inversely proportional, for the smaller preburst luminosity

Table 1: Number of bursts detected from the well known X-ray bursters by BeppoSAX/WFC and INTEGRAL/IBIS/ISGRI and corresponding exposures.

\begin{tabular}{ccccc}
\hline \hline Source & WFC bursts & ISGRI bursts & WFC exposure $(M s)$ & ISGRI exposure $(M s)$ \\
\hline \hline$G X 354-0$ & 423 & 313 & 7.4 & 21.9 \\
$G S 1826-24$ & 260 & 0 & 6.5 & 20.0 \\
$A 1742-294$ & 178 & 0 & 7.0 & 22.3 \\
$4 U 1702-429$ & 104 & 37 & 8.9 & 13.9 \\
$4 U 1705-44$ & 66 & 0 & 8.7 & 12.7 \\
$4 U 1636-536$ & 61 & 8 & 4.7 & 8.4 \\
$G X 3+1$ & 61 & 1 & 6.9 & 22.3 \\
$4 U 1820-30$ & 49 & 0 & 7.1 & 21.5 \\
\hline \hline
\end{tabular}



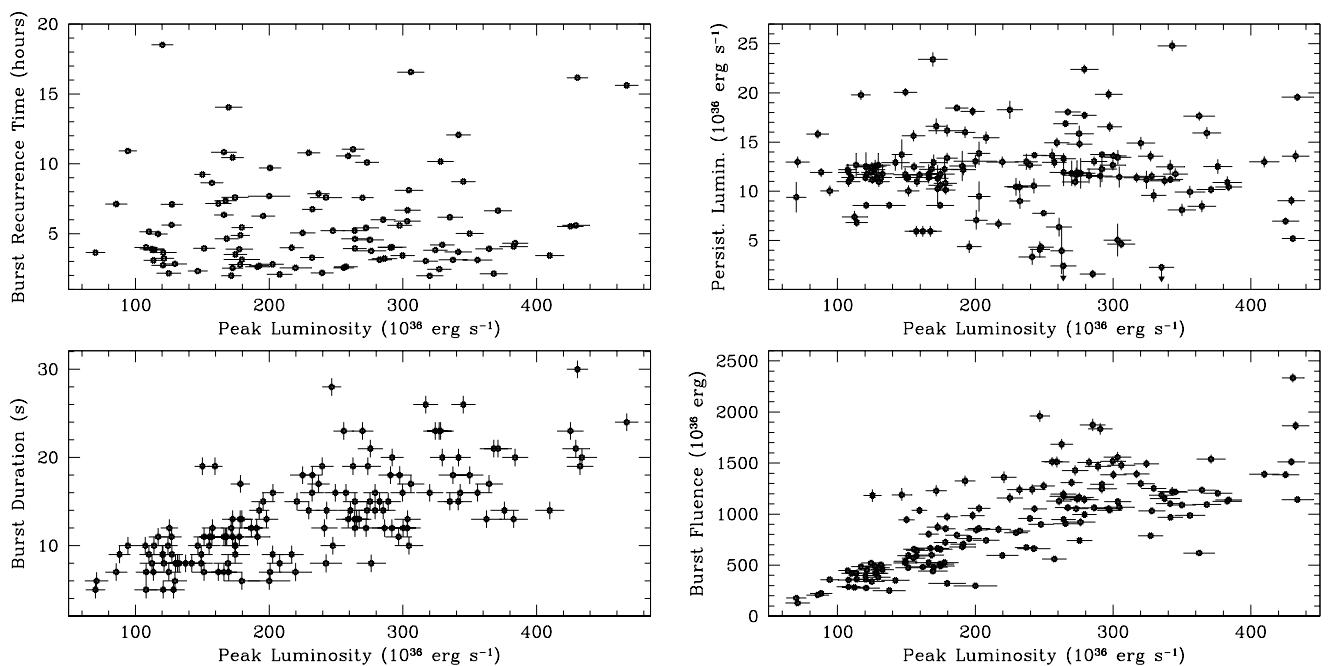

Figure 9: Burst recurrence time (top,left), duration (bottom,left), fluence (bottom,right) and preburst luminosity (top,right) versus burst peak luminosity for 145 bursts, detected from GX 354-0 by JEM-X telescope in 2003-2007.

(i.e. accretion rate) is the longer it takes to accrete enough matter for the next burst. The two lines show this fi tted dependence for consecutive bursts and the ones with a previous burst possibly omitted due to lags in observations.

Finally Fig. 11 (right panel) shows the dependence of the burst rate from LMXBs on their persistent luminosity. Here we took into account all the LMXBs observed with INTEGRAL with a signifi cant exposure. The general raising trend seen here is well explained by the idea, that the larger the preburst luminosity (i.e. accretion rate) is the less time it takes to accumulate enough fuel for the next burst, so the more frequent the bursts are. The steep fall at high luminosities is also
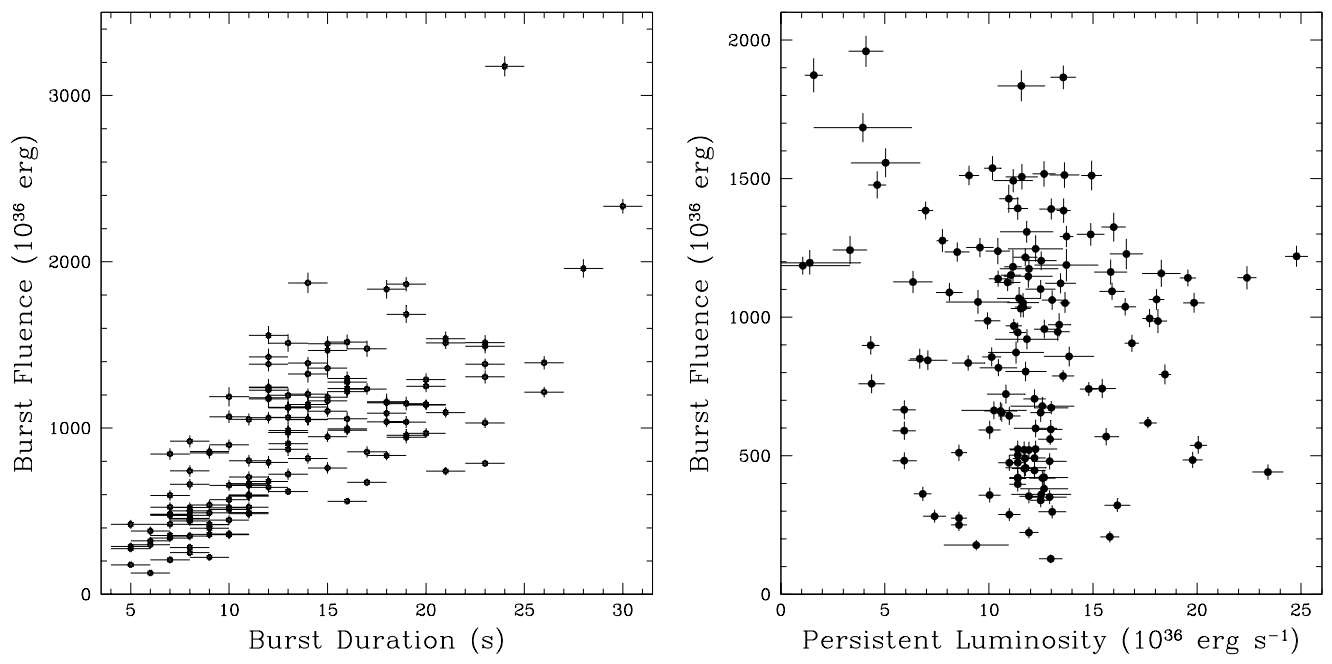

Figure 10: Burst fluence vs. burst duration (left panel) and preburst persistent luminosity (right panel) for 145 bursts detected from GX 354-0 by the JEM-X telescope in 2003-2007. 

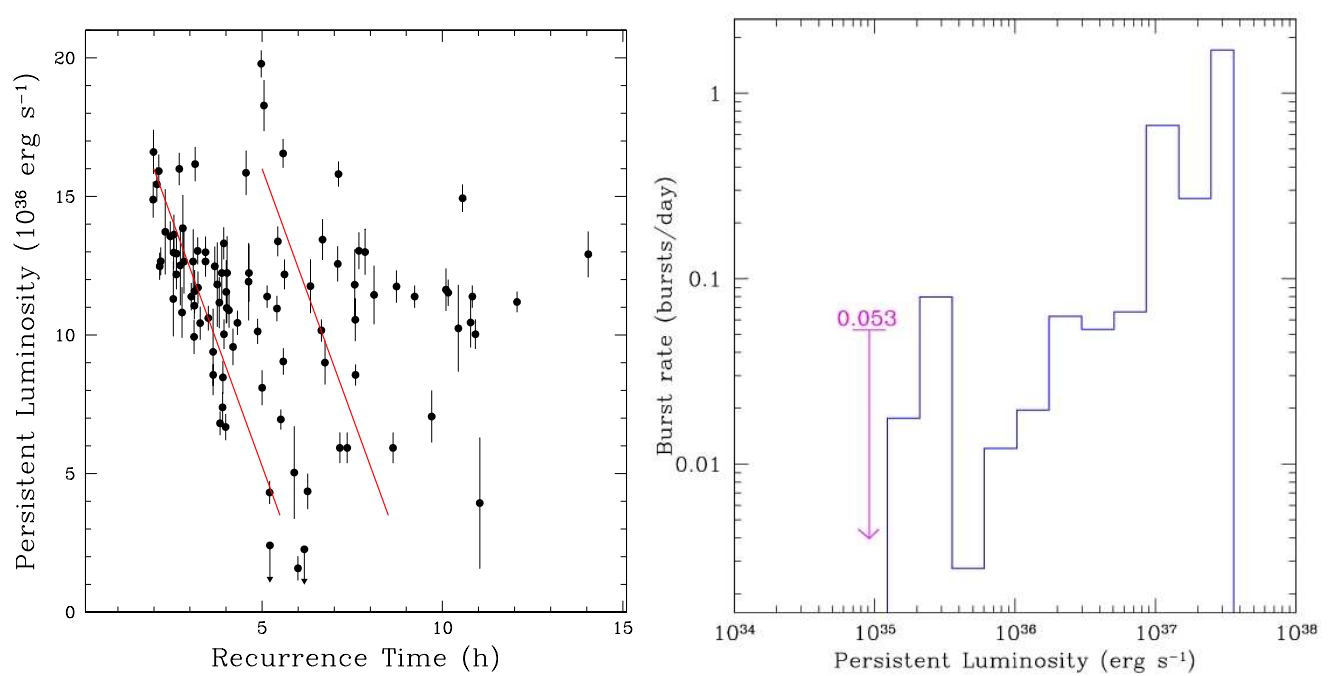

Figure 11: Left panel: Preburst persistent luminosity vs. burst recurrence time for 145 bursts detected from GX 354-0 by both the JEM-X and IBIS telescopes in 2003-2007. Red lines indicate fits to the data, the left one fitting most likely consecutive bursts, and the right one - those with a previous burst possibly omitted due to lags in observations. Right panel: Dependence of the burst rate on the persistent luminosity for all the LMXBs, observed by INTEGRAL with a significant exposure.

well understandable for at some point the thermonuclear burning gets stable and explosions (i.e. bursts) vanish. The most interesting thing here might be the lower luminosity edge. Here we were able to put a $3 \sigma$ upper limit for the burst rate of 0.053 bursts/day from systems with a persistent $\mathrm{X}$-ray luminosity around $10^{35} \mathrm{erg} \mathrm{s}^{-1}$. Further investigation of this area might give some insights and estimations on the peculiarities of bursting behaviour at lower accretion rate levels.

\section{CONCLUSIONS}

As a result of the detailed analysis of all the publicly open data, obtained by the ISGRI detector of the IBIS telescope on-board the INTEGRAL observatory from February 2003 to March 2007, we were able to create a catalog of 424 type I X-ray bursts from 17 known and 2 newly discovered X-ray bursters. One of the byproducts of this study was a discovery of a gamma-ray burst GRB060428C, that was not detected by IBAS.

This work revealed a unique feature of GX 354-0 - many of the bursts from this burster have hard X-ray tails, signifi cantly detectable above $15 \mathrm{keV}$. A detailed analysis of GX 354-0 bursts sample revealed a direct proportionality of burst duration, fuence and peak luminosity.

\section{ACKNOWLEDGMENTS}

Authors are grateful to Mike Revnivtsev for discussion. This work is based on the observational data obtained by INTEGRAL and provided through the Russian and European INTEGRAL Science Data Centers. The study was supported by the Presidium of the Russian Academy of Sciences (the "Origin and Evolution of Stars and Galaxies" project), and the Program for Support of Russian Leading Scientifi c Schools (project no. NSh-5579.2008.2). 


\section{References}

[1] Bildsten, L. 2000, American Institute of Physics Conference Series, 522, 359

[2] Chelovekov, I. V., Grebenev, S. A., \& Sunyaev, R. A. 2006, Astronomy Letters, 32, 456

[3] Chelovekov, I. V., Grebenev, S. A., \& Sunyaev, R. A. 2007, Proceedings of the 6th Integral Workshop, 445 (SP-622)

[4] Chelovekov, I. V., \& Grebenev, S. A. 2007, Astronomy Letters, 33, 807

[5] Cornelisse, R., et al. 2003, Astronomy and Astrophysics, 405, 1033

[6] S. A. Grebenev, M. N.Pavlinsky, \& R. A. Sunyaev, in Proc. of the Conf. "Roentgenstrahlung from the Universe" (Ed. H.U. Zimmermann, J.E. Truemper, H. Yorke, Germany, Wuerzburg, MPE Report 263, 1996), 141

[7] Grebenev, S. A., \& Chelovekov, I. V. 2007, Astronomy Letters, 33, 789

[8] Lewin, W. H. G., van Paradijs, J., \& Taam, R. E. 1993, Space Science Reviews, 62, 223 